\title{
Consequences of conceptus exposure to colony-stimulating factor 2 on survival, elongation, interferon- $\tau$ secretion, and gene expression
}

Barbara Loureiro ${ }^{1}$, Jeremy Block ${ }^{1,2}$, Mauricio G Favoreto ${ }^{1}$, Silvia Carambula ${ }^{1}$, Kathleen A Pennington ${ }^{1}$, Alan D Ealy ${ }^{1}$ and Peter J Hansen ${ }^{1}$

${ }^{1}$ Department of Animal Sciences and D.H. Barron Reproductive and Perinatal Biology Research Program, University of Florida, PO Box 110910, Gainesville, Florida 32611-0910, USA and ${ }^{2}$ Ovatech LLC, Gainesville, Florida 32605, USA

Correspondence should be addressed to P J Hansen; Email: hansen@animal.ufl.edu

Reproduction (2014) 147 X1

The authors apologise for an error in the results reported in this article published in Reproduction (vol 141, pp 617-624). A mathematical error resulted in calculation of values for IFN in uterine flushings that were too high. The corrected results are presented in Figure 1 (published in full below with the corrected figure legend).
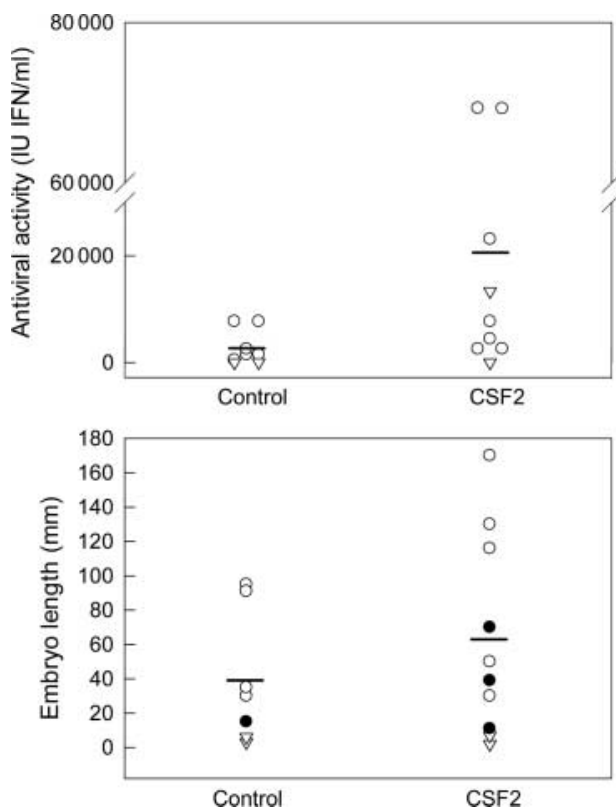

Figure 1 Individual values of antiviral activity in uterine flushings (top) in pregnant cows and length of recovered conceptuses (bottom). Triangles represent tubular conceptuses and circles represent filamentous

conceptuses. In the bottom panel, conceptuses that were not recovered intact are represented by closed circles. The horizontal bars represent the mean value for each treatment. Antiviral activity was not affected by treatment. However, when considering only those cows in which detectable antiviral activity was present, antiviral activity was greater for cows receiving CSF2-treated conceptuses $(P<0.05)$. Conceptus length was not significantly affected by treatment.
Note that the correlation between conceptus length and IFN concentration in uterine flushings was 0.08 and not 0.02 as stated in the paper. The range of IFN concentrations in uterine flushings ranged from nondetectable to almost $70000 \mathrm{IU} / \mathrm{mL}$ (and not the $9000000 \mathrm{U} / \mathrm{ml}$ as stated in the paper). In addition, the concentration of IFN in uterine flushings of the nonpregnantcow with detectable IFN was $494 \mathrm{IU} / \mathrm{ml}$ rather than $59049 \mathrm{IU} / \mathrm{mL}$. For all cows, there was a nonsignificant trend for antiviral activity to be greater for cows receiving CSF2-treated conceptuses than for cows receiving control conceptuses. This difference was $P<0.05$ (rather than the $P=0.07$ as stated in the paper) when only cows with detectable antiviral activity were considered.

The authors regret this error. 